\title{
Prominent autistic traits and subthreshold bipolar/mixed features of depression in severe anorexia nervosa
}

\author{
Michele Fornaro, ${ }^{1}$ iD Teresa Sassi, ${ }^{1}$ Stefano Novello, ${ }^{1}$ Annalisa Anastasia, ${ }^{2}$ Andrea Fusco, ${ }^{1}$ \\ Ignazio Senatore, ${ }^{1}$ Andrea de Bartolomeis ${ }^{1}$ \\ ${ }^{1}$ Dipartimento di Neuroscienze, Sezione di Psichiatria, Università Federico II di Napoli, Italy. ${ }^{2}$ Istituto Nazionale di Previdenza Sociale, Latina, \\ Italy.
}

\begin{abstract}
Objective: Autistic traits are associated with a burdensome clinical presentation of anorexia nervosa (AN), as is AN with concurrent depression. The aim of the present study was to explore the intertwined association between complex psychopathology combining autistic traits, subthreshold bipolarity, and mixed depression among people with AN.

Method: Sixty patients with AN and concurrent major depressive episode (mean age, $22.2 \pm 7$ years) were cross-sectionally assessed using the Autism-Spectrum Quotient test (AQ-test), the Hamilton depression scales for depression and anxiety, the Young Mania Rating Scale (YMRS), the Hypomania-Checklist-32 (HCL-32), second revision (for subthreshold bipolarity), the Brown Assessment and Beliefs Scale (BABS), the Yale-Brown-Cornell Eating Disorders Scale (YBC-EDS), and the Eating Disorder Examination Questionnaire (EDE-Q). Cases were split into two groups depending on body mass index $(\mathrm{BMI})$ : severe $\mathrm{AN}\left(\mathrm{AN}^{+}\right)$if $\mathrm{BMI}<16$, not severe $\left(\mathrm{AN}^{-}\right)$if $\mathrm{BMI} \geqslant 16$.

Results: The "subthreshold bipolarity with prominent autistic traits" pattern correctly classified $83.6 \%$ of $\mathrm{AN}$ patients $\left(\mathrm{AN}^{+}=78.1 \%\right.$; $\left.\mathrm{AN}^{-}=91.3 \%, \operatorname{Exp}(\mathrm{B})=1.391\right)$. $\mathrm{AN}^{+}$cases showed higher rates of positive scores for YMRS items 2 (increased motor activity-energy) and 5 (irritability) compared to $\mathrm{AN}^{-}$ cases.

Conclusions: In our sample, depressed patients with severe AN had more pronounced autistic traits and subtly mixed bipolarity. Further studies with larger samples and prospective follow-up of treatment outcomes are warranted to replicate these findings.
\end{abstract}

Keywords: Anorexia nervosa; autism spectrum; bipolar disorder; mixed depression

\section{Introduction}

Accumulating evidence suggests a relationship between autism spectrum disorders (ASDs) and anorexia nervosa (AN). ${ }^{1-3}$

An underlying genetic vulnerability shared by $\mathrm{AN}$ and ASDs has been proposed since the late 1980s, with research pointing out prominent AN features in (pre-) adolescent females and autistic features in infant males, following the interaction of certain environmental factors. ${ }^{4}$ Elevated autistic traits have also been reported in females with $\mathrm{AN},{ }^{5,6}$ as have morphometric alterations in brain areas linked to social cognition, including the amygdala, the orbitofrontal cortex, the fusiform face area (in the temporal lobe and Brodmann's area 37), and the superior temporal sulcus. ${ }^{7,8}$ Overall, the prevalence of ASD in AN has been reported to approximate $23 \%$ of cases. ${ }^{9}$ This is a compelling finding, taking into account the similarities in cognitive, social, and emotional processing

Correspondence: Michele Fornaro, Università degli Studi di Napoli Federico II, Sezione di Psichiatria, Ospedale Policlinico, via Pansini n.5, edificio 18, Psichiatria, 80131, Napoli, Italy.

E-mail: dott.fornaro@gmail.com

Submitted Mar 28 2019, accepted Jul 22 2019, Epub Nov 042019. between $\mathrm{AN}$ and ASD. ${ }^{10,11}$ These similarities pertain attention-switching tasks, perspective taking and fantasy, lack of emotional introspection, and the theory of mind profile, ${ }^{12}$ and have led to the conceptualization of AN as a distinct female autism phenotype. ${ }^{1}$ The presence of autisticspectrum features in AN is associated with a more severe clinical presentation, depression, anxiety, and poorer everyday functioning overall. ${ }^{13,14}$ Poorer treatment outcomes of AN have been documented in patients with traitlike (stable) ASD features, ${ }^{15}$ possibly leading to multiple treatment-augmentation attempts and polypharmacy. ${ }^{16}$

The impact of ASD on the outcomes of AN treatment deserves research attention, especially considering that ASD is associated with a fourfold increase in lifetime depression rates ${ }^{17}$ and that adults with prominent autistic traits have high rates of suicide attempts. ${ }^{18}$ Little attention has been given to the impact of depression in this particular population, although clinical practice suggests a high burden of depression (often associated with irritability
How to cite this article: Fornaro M, Sassi T, Novello S, Anastasia A, Fusco A, Senatore I, et al. Prominent autistic traits and subthreshold bipolar/mixed features of depression in severe anorexia nervosa. Braz J Psychiatry. 2020;42:153-161. http://dx.doi.org/ 10.1590/1516-4446-2019-0500 
and increased motor-activity/energy expenditure, possibly seen as subtle hints of bipolarity) and prominent autistic traits in AN.

To the best of our knowledge, no research has been published concerning the potential impact of concurrent depression (herein including subthreshold bipolar depression) and autistic traits on body mass index (BMI) scores in AN, a parameter otherwise regarded as a hallmark of severity in the DSM-5. ${ }^{19}$

However, we hypothesize that the presence of autistic traits and subthreshold bipolarity with mixed features may be more pronounced among severe AN people with a current major depressive episode (MDE).

Therefore, the present study aims at preliminary assess the impact of depression and autistic traits on the severity of $\mathrm{AN}$, ideally paving the ground for future prospective studies providing a systematic neuropsychological and treatment-outcome assessment.

\section{Methods}

The procedures adopted in the present cross-sectional study are outlined below, following the recommendations of the Strengthening the Reporting of Observational Studies in Epidemiology (STROBE) statement. ${ }^{20}$

\section{Study setting, participants, and eligibility criteria}

In- and outpatients of both sexes, aged 14 years or older, with a primary diagnosis of $\mathrm{AN}$ and comorbid MDE, either in the course of major depressive disorder (MDD) or bipolar disorder (BD), were screened for inclusion. Given the non-experimental design of this study, the inclusion criteria were very permissive.

Enrollment took place in January 2017 and October 2018, at the outpatient psychiatric unit of Federico II University of Naples, Italy. Two experienced psychiatrists (TS and IS) were appointed to establish the diagnoses according to the DSM-5 criteria. The exclusion criteria were substance use disorder, lack of valid informed consent, and any condition potentially affecting BMI. Finally, AN subjects were split into two groups: severe $\left(\mathrm{AN}^{+}\right)$if $\mathrm{BMI}<16$ or non-severe $\left(\mathrm{AN}^{-}\right)$if $\mathrm{BMI} \geqslant 16$, as per the DSM-5 threshold.

\section{Variables, measures, and procedures}

Once demographic and clinical data had been gathered, a broad range of rating tools were administered. The AutismSpectrum Quotient-test (AQ-test), Italian-language version, ${ }^{21}$ has also been validated for use with patients with a primary diagnosis of $A N{ }^{10}$ The $A Q$-test includes 50 items assessing five areas of functioning: social skill, attention switching, attention to detail, communication, and imagination. The Hamilton Rating Scale for Depression 17-item version (HAM-D-17) ${ }^{22}$ and Anxiety (HAM-A) ${ }^{23}$ and the Young Mania Rating Scale (YMRS) were administered to all participants. Additionally, the Hypomania Checklist-32item, second revision (HCL-32-R2, 34-item version), was used to test for presence of subthreshold bipolarity among participants with a DSM-5 diagnosis of unipolar major depression. The severity and the type of eating disorder were self-reported using the Yale-Brown-Cornell Eating Disorders Scale (YBC-EDS) ${ }^{24}$ and the Eating Disorder Examination Questionnaire (EDE-Q). ${ }^{25}$ The YBS-EDS assess both current and "worst ever" scores for rituals and preoccupations about a range of habits and somatic features. The EDE-Q comprises three domains: restraint, eating concern, and weight concern. Finally, a multipurpose scale for the assessment of insight of illness, the Brown Assessment and Beliefs Scale (BABS), ${ }^{26}$ was administered.

On all of the aforementioned scales, higher scores indicate a higher symptom burden. Patients' clinical records were anonymized and pooled for the present analysis.

\section{Statistical analysis}

All statistical analyses were performed in SPSS version 25.0 for Windows, adopting an alpha error rate of 0.05 (two-tailed) and a conservative statistical power of $95 \%$. Interrater reliability for the analysis was ascertained preliminarily using Cohen's $\kappa$ statistic, to ensure consistency of clinician-administered ratings among raters and minimize rating bias. The normality of data distribution and the homogeneity of variance were verified using the Kolmogorov-Smirnov and Levene tests, respectively. Parametric comparative analyses for demographic and clinical characteristics of the groups were performed using an independent-samples Student $t$-test for continuous variables where appropriate (with the Wilcoxon rank-sum test planned for nonparametric distributions) and chi-square analysis for categorical variables (again, nonparametric as appropriate). Pearson's or Spearman's rho correlations were calculated for selected clinical variables as appropriate. Binary logistic regression (dependent variable $=\mathrm{AN}^{+} / \mathrm{AN}^{-}$) was carried out using the conditional backward logistic regression method for those variables having a significantly different distribution between the $\mathrm{AN}^{+}$vs. $\mathrm{AN}^{-}$groups upon descriptive analysis; linear regression analysis using $\mathrm{BMI}$ as a dependent variable followed the logistic regression. Finally, missing data were excluded pairwise.

\section{Ethics statement}

The participating patients provided a valid, written informed consent after two experienced psychiatrists (TS and IS) had thoroughly explained the study procedures, according to the norms outlined by the local ethics committee.

\section{Results}

Among 72 potentially eligible patients screened, 60 were confirmed eligible for inclusion. Of these, 56 had MDD; three had BD-II (two $\mathrm{AN}^{+}$and one $\left.\mathrm{AN}^{-}\right)$; and one $\left(\mathrm{AN}^{+}\right)$ had BD-I. Excluded patients either failed to meet the diagnostic criteria of a current MDE $(n=10)$ or refused consent due to privacy concerns $(n=2)$.

The sample included 55 females $(F)$ and five males (M). Overall, 35 patients were rated as $\mathrm{AN}^{+}(\mathrm{F} / \mathrm{M}=31 / 4)$ 
and 25 as $\mathrm{AN}^{-}(\mathrm{F} / \mathrm{M}=24 / 1)$. The age of the $\mathrm{AN}^{+}$patients ranged from 14-46 years (mean \pm standard deviation $[S D]=22.82 \pm 6.99$ ); 26 were adult (aged 18 years or older). In the $\mathrm{AN}^{-}$group, age ranged between $15-45$ years (mean $\pm \mathrm{SD}=21.32 \pm 7.16$ ), of whom 18 were adult. Most $\mathrm{AN}^{+}$cases were inpatients at the time of assessment $(27$ of $35,77.1 \%$ ), in contrast to the $\mathrm{AN}^{-}$cases (6 of 25 , or $24 \%$ of cases). Additional demographic and clinical features are described in Table 1.

\section{Interrater variability}

The preliminary interrater variability for diagnosis of current MDE (HAM-D-17 total score $\geqslant 7$ ) was $\kappa=0.749$ $(p \leqslant 0.001), 95 \%$ confidence interval $(95 \% \mathrm{Cl})-0.0710$ to 0.1038 , denoting substantial agreement. Additional measures of interrater variability for other non-self-report ratings ranged from 0.72 (substantial agreement) to 0.83 (almost perfect agreement).

\section{Selected correlation measures in the $A N^{+}$group}

Figure 1 provides a pictorial synthesis of the correlation between $\mathrm{BMI}$ and YMRS total scores in the $\mathrm{AN}^{+}$and $\mathrm{AN}^{-}$ groups.

Essential correlation metrics in the $\mathrm{AN}^{+}$and $\mathrm{AN}^{-}$groups are reported in Table 2.

YMRS items 2 (increased motor activity-energy) and 5 (irritability) correlated significantly with $\mathrm{AN}^{+}: \mathrm{rs}=0.452$ $(p \leqslant 0.001)$ and $r s=0.406(p \leqslant 0.001)$, respectively. YMRS item 5 also correlated with AQ-test total score in AN: $r s=0.353(p \leqslant 0.001)$. The strongest and most significant (positive) correlation in the AQ-test subset was found between AQ-test attention switching and EDE-Q shape, in the $\mathrm{AN}^{+}$group only $(r=0.492, \mathrm{p} \leqslant 0.001)$.

\section{Essential multivariate statistics}

A backward (conditional) stepwise binary logistic regression analysis was conducted to allocate patients to either the $\mathrm{AN}^{+}$or the $\mathrm{AN}^{-}$group, accounting for autistic traits measured using the AQ-test scale and subthreshold bipolarity scores recorded with the HCL-32-R2, among other predictors.

A test of the full model against a constant-only model was statistically significant, indicating that the predictors as a set reliably discriminated between $\mathrm{AN}^{+/-}$cases: $\left(\chi^{2}{ }_{[2]}=27.409 ; p \leqslant 0.001\right)$. Nagelkerke's $R^{2}=0.528$ indicated a sound explanatory value of the regression model. Overall AN predictive capacity was $83.6 \%\left(\mathrm{AN}^{+}=78.1 \%\right.$; $\mathrm{AN}^{-}=91.3 \%$ ).

Additional variables were likewise included in the multistep procedure outlined in Table 3, although step 6 of the logistic regression (AQ-test and HCL-32-R2 total scores input as covariates) provided the best fit, possibly due to multicollinearity issues as well. The Wald criterion demonstrated that both $A Q$-test total $(p=0.039)$ and HCL-32-R2 total ( $p=0.001$ ) made significant contributions to the prediction, with prominent autistic traits and higher HCL-32-R2 scores providing odd ratios (OR) $(\operatorname{Exp}[$ betas] $)$ - of roughly 1.2 and 1.4 , respectively, for prediction of $\mathrm{AN}^{+}$vs. $\mathrm{AN}^{-}$categorization. For purposes of convenience and ease of interpretation, an $\mathrm{OR}=1$ indicates the same propensity of an event belonging to a given AN subgroup.

Linear regression analysis using $\mathrm{BMI}$ total score as the dependent variable and AQ-test and HCL-32-R2 total scores as covariates indicated that the model predicted the BMI scores of AN patients (adjusted $\mathrm{R}^{2}=$ $0.383 ; p \leqslant 0.001$ ). However, only the standardized $b$ coefficient of $A Q$-test was significant $(p \leqslant 0.001)$.

\section{Discussion}

The primary aim of the present study was to explore severe AN through correlations with complex psychopathology (combining autistic traits, mixed features, and subthreshold bipolarity) among patients presenting with a MDE. As expected, descriptive analyses indicated that $\mathrm{AN}^{+}$cases were more frequently inpatients, on pharmacological treatment (especially benzodiazepines or antipsychotics), had higher rates of lifetime psychiatric hospitalization, and had significantly higher scores on all domains of the YBC-EDS and global EDE-Q compared to $\mathrm{AN}^{-}$. Consistent with previous research, ${ }^{10-12}$ the preliminary results of the present study indicate that severe AN is associated with higher scores on the AQ-test compared to $\mathrm{AN}^{-}$, especially in the attention-switching and communication domains.

Multiple lines of research support the connection between ASD and AN, including shared neurobiology 7,8 and genome-wide association studies. ${ }^{27}$ Elevated ASD traits are associated with lower BMI scores, suggesting that ASD traits may arise from the starved state of $A^{28}$ and thus warrant follow-up over the natural history and treatment course of AN. The relationship between AN and ASD is nonspecific, so that ASD traits could be overrepresented in AN, perhaps even maintaining the associated eating disorder rather than causing it. ${ }^{13}$ It has been hypothesized that rigidity, obsessive interest, and social withdrawal/impairments could be the manifestations of an underlying ASD or may be the consequences of highly prevalent conditions in AN, such as starvation, anxiety, depression, and obsessive-compulsive disorder. ${ }^{29}$ The label of "severe" AN proposed by the DSM- 5 based solely on BMI, as we adopted in the present study, may therefore insufficient to capture the complexity of the phenomenon of $\mathrm{AN}$, especially when combined with prominent autistic traits and depression, both of which may have a pathoplastic effect of AN. Similarly, very low BMI could induce agitation, hyperactivity, problematic physical activity $^{30}$ and other bipolar-like symptoms through anaerobic (ketotic) metabolism; thus, we cannot rule out that at least part of the psychopathological features associated with $\mathrm{AN}^{+}$could be explained as "soft bipolarity" due to severe metabolic disturbances and malnutrition, which is a condition known to predict inadequate response towards standalone antidepressant drugs ${ }^{31}$ (indeed, roughly half of the $\mathrm{AN}^{+}$patients in our sample were on antidepressants). If corroborated by prospective follow-up, changes in $\mathrm{BMI}$ scores in the $\mathrm{AN}^{+}$subgroup may explain at least part of the bipolar-like and autism-spectrum symptoms 
Table 1 Demographic and clinical features of the subjects included in the study

\begin{tabular}{|c|c|c|c|c|}
\hline Study subjects $(n=60)$ & Severe AN n=35 (58\%) & Non-severe AN n=25 (42\%) & $t$ or $\chi^{2}(\mathrm{df})$ & $\mathrm{p}$-value \\
\hline \multicolumn{5}{|l|}{ Demographics } \\
\hline Age in years, mean $\pm S D$ (range) & $22.82 \pm 6.99(14-46)$ & $21.32 \pm 7.16(15-45)$ & $-0.816_{(58)}$ & ns \\
\hline Female-to-male ratio, $\mathrm{n}(\%)$ & $31(88.6) / 4(11.4)$ & $24(96.0) / 1(4.0)$ & $1.054_{(1)}$ & ns \\
\hline \multicolumn{5}{|l|}{ Education level } \\
\hline Primary & $1(2.9)$ & $0(0.0)$ & $5.362_{(3)}$ & ns \\
\hline Secondary & $16(45.7)$ & $19(72.0)$ & & \\
\hline Higher & $16(45.7)$ & $5(20.0)$ & & \\
\hline College or university & $2(5.7)$ & $2(8.0)$ & & \\
\hline \multicolumn{5}{|l|}{ Clinical features } \\
\hline BMI & $14.07 \pm 1.44$ & $17.12 \pm 0.91$ & $9.334_{(58)}$ & $<0.001$ \\
\hline Duration of untreated illness, years (mean \pm SD) & $5.68 \pm 4.17$ & $4.08 \pm 6.05$ & $-1.161_{(58)}$ & ns \\
\hline Age at onset, years (mean $\pm S D$ ) & $17.2 \pm 6.22$ & $17.24 \pm 4.78$ & $-0.027_{(58)}$ & ns \\
\hline Current MDE & $35(100.0)$ & $25(100.0)$ & & ns \\
\hline Current mixed features (DSM-5 defined) & $16(45.7)$ & $4(16.0)$ & $5.794_{(1)}$ & 0.016 \\
\hline Purging type & $9(25.7)$ & $6(24.0)$ & $0.023_{(1)}$ & ns \\
\hline Lifetime MDD & $32(91.4)$ & $24(96.0)$ & $0.490_{(1)}$ & ns \\
\hline Lifetime BD-II & $2(5.7)$ & $1(4.0)$ & $0.090_{(1)}$ & ns \\
\hline Lifetime BD-I & $1(2.9)$ & $0(0.0)$ & $0.726_{(1)}$ & ns \\
\hline Lifetime OCD & $5(14.3)$ & $2(8.0)$ & $0.559_{(1)}$ & ns \\
\hline Lifetime anxiety disorders other than OCD & $10(28.6)$ & $5(20.0)$ & $0.571_{(1)}$ & ns \\
\hline Lifetime BN & $7(20.0)$ & $5(20.0)$ & $0.000_{(1)}$ & ns \\
\hline Lifetime BED & $3(8.6)$ & $0(0.0)$ & $2.256_{(1)}$ & ns \\
\hline Lifetime suicidal behavior & $6(17.1)$ & $3(12.0)$ & $0.303_{(1)}$ & ns \\
\hline Lifetime hospitalization & $17(48.6)$ & $3(12.0)$ & $8.777_{(1)}$ & 0.003 \\
\hline Currently on pharmacotherapy (any type) & $31(88.6)$ & $12(48.0)$ & $11.822_{(1)}$ & 0.001 \\
\hline Currently on antidepressant & $16(45.7)$ & $9(36.0)$ & $0.566_{(1)}$ & ns \\
\hline Currently on benzodiazepines & $21(60.0)$ & $6(24.0)$ & $7.636_{(1)}$ & 0.006 \\
\hline Currently on second-generation antipsychotic & $14(40.0)$ & $1(4.0)$ & $10.08(1)$ & 0.001 \\
\hline Currently on lithium & $0(0.0)$ & $1(4.0)$ & $1.42(1)$ & ns \\
\hline Currently on other mood stabilizer & $1(2.9)$ & $4(16.0)$ & 3.29 & ns \\
\hline Family history of eating disorders & $3(8.6)$ & $1(4.0)$ & $0.49_{(1)}$ & ns \\
\hline Family history of affective disorders & $10(28.6)$ & $8(32.0)$ & $0.082(1)$ & ns \\
\hline Family history of anxiety disorders other than OCD & $11(31.4)$ & $6(24.0)$ & $0.396(1)$ & ns \\
\hline Family history of suicidal behavior & $0(0.0)^{\prime}$ & $0(0.0)$ & & ns \\
\hline Family history of OCD & $3(8.6)$ & $1(4.0)$ & $0.490_{(1)}$ & ns \\
\hline Inpatient status & $27(77.1)$ & $6(24.0)$ & $16.641_{(1)}$ & $<0.001$ \\
\hline Number of previous hospitalizations (mean \pm SD) & $0.74 \pm 1$ & $0.2 \pm 0.5$ & $-2.416_{(58)}$ & 0.019 \\
\hline AQ-test total score (mean $\pm S D)$ & $24.25 \pm 5.29$ & $20.04 \pm 4.46$ & $-3.241_{(58)}$ & 0.002 \\
\hline Attention switching & $7.11 \pm 1.07$ & $5.32 \pm 1.51$ & $-5.35(58)$ & $<0.001$ \\
\hline Attention to detail & $3.48 \pm 1.24$ & $3.40 \pm 1.19$ & $-0.268_{(58)}$ & ns \\
\hline Communication & $3.94 \pm 1.58$ & $3 \pm 1.08$ & $-2.570_{(58)}$ & 0.013 \\
\hline Imagination & $3.08 \pm 1.19$ & $3.94 \pm 1.58$ & $-2.570_{(58)}$ & ns \\
\hline Social skills & $6.62 \pm 1.68$ & $5.88 \pm 1.3$ & $-1.861_{(58)}$ & ns \\
\hline ADHD & $2(5.7)$ & $0(0.0)$ & $1.478_{(1)}$ & ns \\
\hline HCL-32-R2 total score* (mean $\pm \mathrm{SD})$ & $7.78 \pm 5.05$ & $4.34 \pm 3.94$ & $-2.714_{(58)}$ & 0.009 \\
\hline \multicolumn{5}{|l|}{ Rating scales } \\
\hline HAM-D-17, total score (mean \pm SD) & $17.74 \pm 5.19$ & $13.4 \pm 3.35$ & $-3.663_{(58)}$ & 0.001 \\
\hline YMRS, total score (mean \pm SD) & $7.51 \pm 4.04$ & $3.4 \pm 2.56$ & $-4.476(58)$ & $<0.001$ \\
\hline $\begin{array}{l}\text { YMRS, item } 2 \text { (increased motor activity-energy) recoded } \\
\text { as yes/no }\end{array}$ & $23(65.7)$ & $5(20.0)$ & $12.245_{(1)}$ & $<0.001$ \\
\hline YMRS, item 5 (irritability) recoded as yes/no & $29(89.2)$ & $11(44.0)$ & $9.909_{(1)}$ & 0.002 \\
\hline $\begin{array}{l}\text { YMRS, item } 7 \text { (language-thought disorder) recoded as } \\
\text { yes/no }\end{array}$ & $8(22.9)$ & $5(20.0)$ & $0.070_{(1)}$ & ns \\
\hline $\begin{array}{l}\text { YMRS, item } 9 \text { (disruptive-aggressive behavior) recoded } \\
\text { as yes/no }\end{array}$ & $8(22.9)$ & $3(12.0)$ & $1.148_{(1)}$ & ns \\
\hline HAM-A total score $($ mean \pm SD) & $13.34 \pm 4.23$ & $10.88 \pm 3.19$ & $-2.45_{(58)}$ & 0.017 \\
\hline BABS total score (mean \pm SD) & $8.51 \pm 4.69$ & $8.12 \pm 5.55$ & $-0.297_{(58)}$ & ns \\
\hline \multicolumn{5}{|l|}{ YBC-EDS (mean \pm SD) } \\
\hline Total, current & $33.85 \pm 5.32$ & $29.64 \pm 5.16$ & $-3.096_{(58)}$ & 0.003 \\
\hline Total, worst & $34.62 \pm 5$ & $30.76 \pm 4.42$ & $-3.06(58)$ & 0.003 \\
\hline Total, current rituals & $11.05 \pm 1.89$ & $9.44 \pm 1.70$ & $-3.394_{(58)}$ & 0.001 \\
\hline Total, worst rituals & $11.25 \pm 1.8$ & $9.84 \pm 1.65$ & $-3.106_{(58)}$ & 0.003 \\
\hline Total, current preoccupations & $11.68 \pm 1.77$ & $9.8 \pm 1.89$ & $-3.94(58)$ & $<0.001$ \\
\hline Total, worst preoccupations & $11.88 \pm 1.71$ & $10.12 \pm 2.04$ & $-3.63_{(58)}$ & 0.001 \\
\hline
\end{tabular}


Table 1 (continued)

\begin{tabular}{|c|c|c|c|c|}
\hline Study subjects $(n=60)$ & Severe AN n=35 (58\%) & Non-severe AN n=25 (42\%) & $t$ or $\chi^{2}(\mathrm{df})$ & $\mathrm{p}$-value \\
\hline \multicolumn{5}{|l|}{ EDE-Q (mean $\pm \mathrm{SD})$} \\
\hline Restraint subscale & $22.4 \pm 3.79$ & $19.52 \pm 5.5$ & $2.401_{(58)}$ & 0.020 \\
\hline Eating concern subscale & $16.65 \pm 4.57$ & $14.48 \pm 3.88$ & $-1.932_{(58)}^{(00)}$ & ns \\
\hline Shape concern subscale & $28.28 \pm 5.67$ & $27.56 \pm 8.23$ & $-0.373_{(58)}$ & ns \\
\hline Weight concern subscale & $15.77 \pm 4.62$ & $16.28 \pm 5.67$ & 0.382 & ns \\
\hline Global & $78.14 \pm 14.28$ & $75.44 \pm 19.43$ & $-2.405_{(58)}$ & 0.019 \\
\hline
\end{tabular}

Data presented as $\mathrm{n}(\%)$, unless otherwise specified.

$\mathrm{ADHD}=$ attention deficit and hyperactivity disorder; $\mathrm{AN}=$ anorexia nervosa; $\mathrm{AQ}$-test $=$ Autism-Spectrum Quotient test; $\mathrm{BABS}=\mathrm{Brown}$ Assessment and Beliefs Scale; $\mathrm{BD}=$ bipolar disorder; $\mathrm{BED}=$ binge eating disorder; $\mathrm{BMI}=$ body mass index; $\mathrm{BN}=$ bulimia nervosa; $\mathrm{df}=$ degrees of freedom; EDE-Q = Eating Disorder Examination Questionnaire; HAM-A = Hamilton Anxiety Rating Scale; HAM-D-17 = Hamilton Scale for Depression, 17-item; HCL-32-R2 = Hypomania Checklist, 32-item, second revision, 34-item version; MDD = major depressive disorder; $\mathrm{MDE}=$ major depressive episode; $\mathrm{ns}=$ nonsignificant; $\mathrm{OCD}=$ obsessive-compulsive disorder; $\mathrm{SD}=\mathrm{standard}$ deviation; $\mathrm{YBC}$-EDS = Yale-Brown-Cornell Eating Disorders Scale; YMRS = Young Mania Rating Scale.

* 56/60 MDD patients.

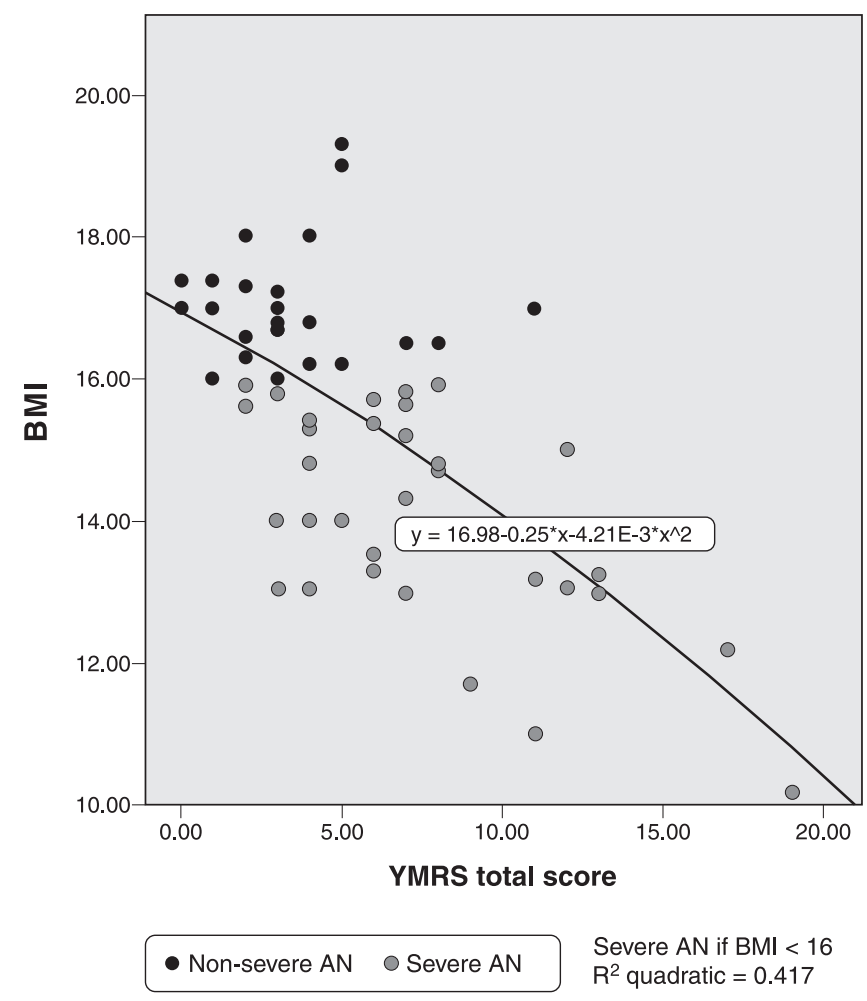

Figure 1 Pearson's correlation of BMI and YMRS total score, $r^{2}=-0.545 ; p \leqslant 0.001$. BMI dichotomizes AN as severe ( $<16$ ) or non-severe $(\geqslant 16)$. This plot provides an estimate of the impact of manic-series symptoms in depression, and should be considered a crude approximation of the impact of the "mixed features" DSM-5 specifier for MDE. AN = anorexia nervosa; $\mathrm{BMI}=$ body mass index; MDE = major depressive episode; YMRS = Young Mania Rating Scale.

upon improvement of imbalances due to anaerobic metabolism and vigorous physical exercise. However preliminary, the results of the present study suggest that DSM-5 mixed features of depression were significant more prevalent among $\mathrm{AN}^{+}$vs. $\mathrm{AN}^{-}$cases, regardless of the putative causes. Furthermore, increased motoractivity/energy and irritability were also more common among those AN individuals with lower BMI scores, although not significantly so. This has major clinical implications, considering that neither "increased motoractivity/energy" nor "irritability" are included by the DSM5 among the mixed features, despite their discriminant predictive value for bipolarity in the clinical setting. ${ }^{32}$
Likewise, the higher HCL-32-R2 scores detected do not necessarily indicate bipolarity in $\mathrm{AN}^{+}$cases, since in most instances the usual cutoff of 14 was not met. As the HCL-32-R2 has not yet been validated for use in patients with DSM-5-defined unipolar depression associated with AN and prominent autistic traits, we submit that even a trend towards higher HCL-32-R2 scores (significantly higher in $\mathrm{AN}^{+}$vs. $\mathrm{AN}^{-}$cases) may indicate a plausible trend toward subthreshold bipolarity (once again, regardless of putative neuroendocrine causes). This is particularly worth of note considering that the potential presence of "signs of excitement" may account for a specific therapeutic approach, assuming an internal medicine workup 


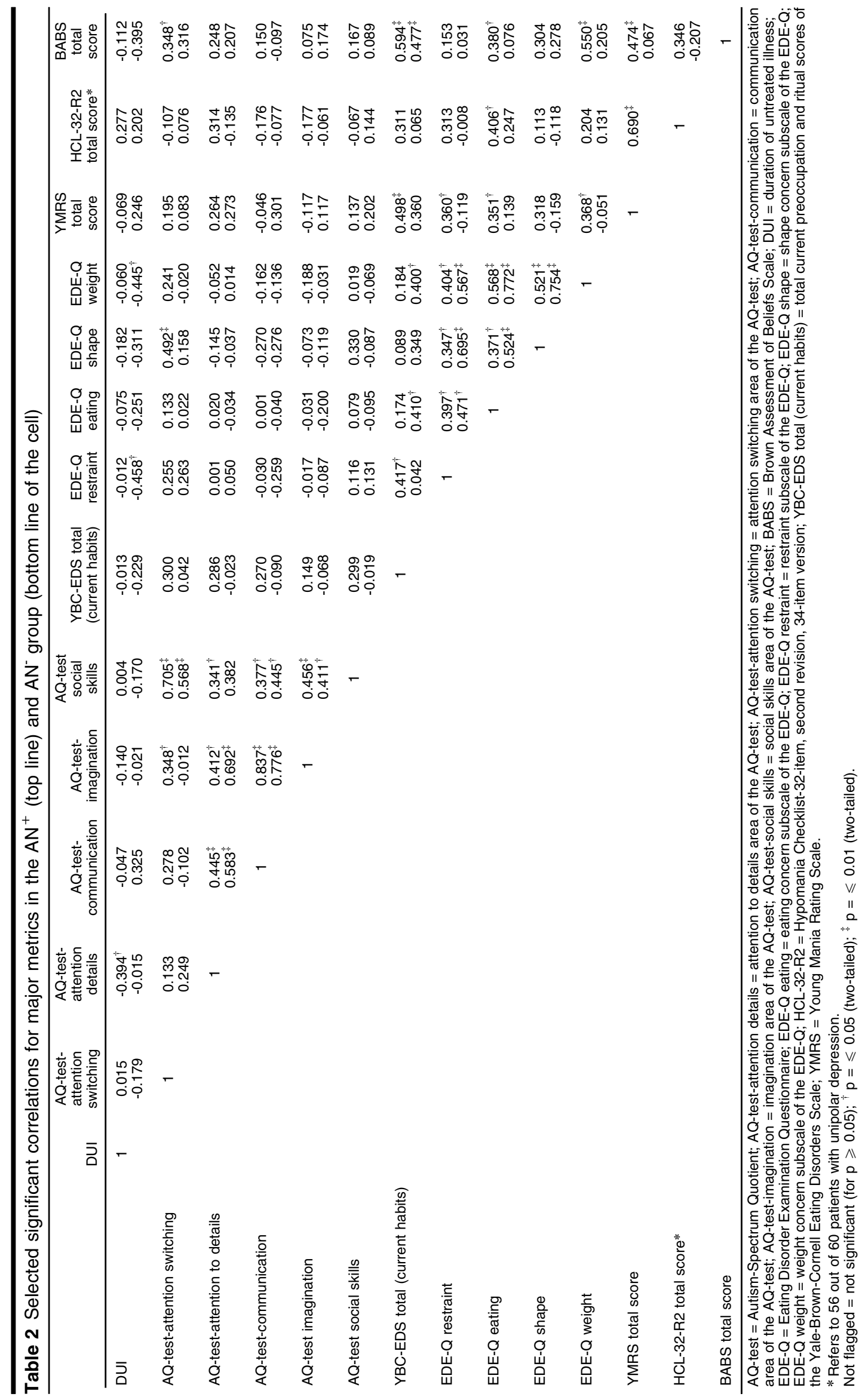


Table 3 Multistep logistic regression

\begin{tabular}{|c|c|c|c|c|c|}
\hline \multirow[b]{2}{*}{ Variables in the equation } & \multirow[b]{2}{*}{ B } & \multirow[b]{2}{*}{$\mathrm{p}$-value } & \multirow[b]{2}{*}{$\operatorname{Exp}(B)$} & \multicolumn{2}{|c|}{$95 \% \mathrm{Cl}$ for $\operatorname{Exp}(\mathrm{B})$} \\
\hline & & & & Lower bound & Upper bound \\
\hline \multicolumn{6}{|l|}{ Step 1} \\
\hline AQ-test total score & 0.198 & 0.027 & 1.219 & 1.023 & 1.454 \\
\hline HCL-32 total score & 0.366 & 0.016 & 1.442 & 1.071 & 1.940 \\
\hline \multicolumn{6}{|l|}{ YMRS } \\
\hline Item 2 & 1.637 & 0.095 & 5.137 & 0.754 & 35.002 \\
\hline Item 5 & 0.267 & 0.780 & 1.306 & 0.201 & 8.497 \\
\hline Item 7 & -1.779 & 0.153 & 0.169 & 0.015 & 1.937 \\
\hline Item 9 & -0.743 & 0.595 & 0.476 & 0.031 & 7.386 \\
\hline BABS total score & -0.119 & 0.189 & 0.888 & 0.744 & 1.060 \\
\hline Constant & -5.584 & 0.004 & 0.004 & & \\
\hline \multicolumn{6}{|l|}{ Step 2} \\
\hline AQ-test total score & 0.202 & 0.023 & 1.224 & 1.028 & 1.456 \\
\hline HCL-32 total score & 0.377 & 0.012 & 1.458 & 1.086 & 1.956 \\
\hline \multicolumn{6}{|l|}{ YMRS } \\
\hline Item 2 & 1.705 & 0.071 & 5.503 & 0.865 & 35.008 \\
\hline Item 7 & -1.754 & 0.156 & 0.173 & 0.015 & 1.958 \\
\hline Item 9 & -0.850 & 0.529 & 0.427 & 0.030 & 6.045 \\
\hline BABS total score & -0.117 & 0.192 & 0.889 & 0.745 & 1.061 \\
\hline Constant & -5.557 & 0.004 & 0.004 & & \\
\hline \multicolumn{6}{|l|}{ Step 3} \\
\hline AQ-test total score & 0.207 & 0.019 & 1.230 & 1.035 & 1.461 \\
\hline HCL-32 total score & 0.330 & 0.007 & 1.392 & 1.094 & 1.770 \\
\hline \multicolumn{6}{|l|}{ YMRS } \\
\hline Item 2 & 1.748 & 0.064 & 5.745 & 0.905 & 36.487 \\
\hline Item 7 & -1.682 & 0.171 & 0.186 & 0.017 & 2.071 \\
\hline BABS total score & -0.123 & 0.173 & 0.885 & 0.741 & 1.055 \\
\hline Constant & -5.543 & 0.004 & 0.004 & & \\
\hline \multicolumn{6}{|l|}{ Step 4} \\
\hline AQ-test total score & 0.166 & 0.041 & 1.180 & 1.007 & 1.383 \\
\hline HCL-32 total score & 0.294 & 0.009 & 1.342 & 1.077 & 1.673 \\
\hline \multicolumn{6}{|l|}{ YMRS } \\
\hline Item 2 & 1.656 & 0.075 & 5.238 & 0.845 & 32.478 \\
\hline Item 7 & -1.441 & 0.235 & 0.237 & 0.022 & 2.551 \\
\hline Constant & -5.375 & 0.004 & 0.005 & & \\
\hline \multicolumn{6}{|l|}{ Step 5} \\
\hline AQ-test total score & 0.172 & 0.031 & 1.188 & 1.016 & 1.390 \\
\hline HCL-32 total score & 0.287 & 0.011 & 1.332 & 1.067 & 1.663 \\
\hline YMRS item 2 & 1.242 & 0.128 & 3.463 & 0.698 & 17.177 \\
\hline Constant & -5.558 & 0.003 & 0.004 & & \\
\hline \multicolumn{6}{|l|}{ Step 6} \\
\hline AQ-test total score & 0.165 & 0.039 & 1.179 & 1.008 & 1.380 \\
\hline HCL-32 total score & 0.347 & 0.001 & 1.415 & 1.146 & 1.748 \\
\hline Constant & -5.228 & 0.004 & 0.005 & & \\
\hline
\end{tabular}

Step 6 provided the best fit, correctly classifying up to $78.1 \%$ of $\mathrm{AN}^{+}$cases.

The HCL-32-R2 scores refer to 56/60 unipolar depression patients.

$95 \% \mathrm{Cl}=95 \%$ confidence interval; AN = anorexia nervosa; AQ-test $=$ Autism-Spectrum Quotient-test; BABS = Brown Assessment of Beliefs Scale; HCL-32 = Hypomania Checklist-32-item, second revision, 34-item version; YMRS = Young Mania Rating Scale.

has already ruled out any underlying organic condition which might affect attitudes toward eating. We hypothesize that, at least in a proportion of severe cases of AN, the combination of prominent autistic traits and depression with irritability and increased motor-activity/energy, alongside obsessive interests (i.e., a focus on shape concerns) and an inability to divert attention switching toward objects/goals other than eating and weight concerns, may represent an atypical mixed state. According to Emil Kraepelin and his pupil Wilhelm Weygandt, depression may accompany "psychomotor activity" and "formal reasoning" in different ways, leading to multiple mixed configurations. ${ }^{33}$ Possibly, at least some depressed $\mathrm{AN}^{+}$ patients may thus present with atypical manifestations of two Kraepelinian mixed states, namely "depressive-anxious mania" (low mood, increased psychomotor activity, and increased formal reasoning) and "aroused depression" (low mood, increased psychomotor activity, and reduced formal reasoning). Alternatively, very severe AN with prominent autistic traits may resemble Esquirol's monomania, a sort of "partial insanity" conceived as "single pathological preoccupation in an otherwise sound mind" 34 (herein, the goal-directed activity/hyperactivity is focused on shape and diet). On the other hand, at least a proportion of 
patients with overt BD may experience lifetime weight cycling (possibly alternating bulimic and anorexic phases) which resemble manic-depressive excitement (vigorous physical exercise and starvation, or intense binging/ purging), alternating with apparent remission of this excitement, resulting in less disabling - yet transient eating disturbances. ${ }^{35,36}$

Ideally, future controlled trials should consider low-dose lithium (upon careful evaluation of cardiac and renal function) and/or antipsychotics (preferably agents with a low propensity to cause cardiac arrhythmias and weight gain, so as to minimize the subsequent risk of refeeding syndrome) for the most severe cases of AN with subthreshold bipolar depression and/or psychotic features, such as lack of insight of illness corroborated by high BABS scores. If confirmed in replication studies including prospective assessment of different treatment modalities, the potential impact of subtle features associated with some of the most severe cases of AN should affect treatment response, as postulated for some MDD cases which are devoid of AN comorbidity yet respond inadequately to medication due to subthreshold bipolarity. ${ }^{37-39}$ Nevertheless, these considerations should be made only after first-line evidence-based treatments, ${ }^{40}$ including intensive medical and psychological care, ${ }^{41,42}$ prove to be ineffective.

Limitations of this study include the small sample size, lack of neuropsychological/IQ testing, and absence of recording of treatment outcomes, energy expenditure, and exercise activity across different treatment interventions. In addition, besides the statistical significance of the corresponding $p$-values, the ORs for the pivotal predictors "autistic traits" (OR = 1.18) and "sub-threshold bipolar depression" (OR $=1.34)$ only slightly exceed 1 , meaning that the association between such predictors and $\mathrm{AN}^{+}$is not definitive (refer to the last step of the regression model in Table 3). The severity of AN was defined solely by BMI threshold, as in the DSM-5. Frail patients with AN may have presented with medical symptoms resembling depression, and no AN control without depression was included in the present study. Female cases were overrepresented, although this reflects the natural history of the condition itself. On the other hand, the "real-world" setting of the present study may have reduced the chances of Berkson's bias.

In conclusion, the findings of the present study must be considered as explorative. No recommendation whatsoever about therapeutic choices can be inferred at this time; any potential implications for treatment must be viewed as a critical, yet theoretical, appraisal, relevant only for forthcoming research on the topic. However, considering the burden related to the complex clinical phenotype of severe AN with comorbid depression and prominent autistic traits and the absence of evidence on the matter, even results warranting replication - such as ours - should be reported in such a hard-to-capture special population. ${ }^{43}$

\section{Disclosure}

The authors report no conflicts of interest.

\section{References}

1 Westwood H, Tchanturia K. Autism spectrum disorder in anorexia nervosa: an updated literature review. Curr Psychiatry Rep. 2017; 19:41.

2 Odent M. Autism and anorexia nervosa: Two facets of the same disease? Med Hypotheses. 2010;75:79-81.

3 Zhou ZC, McAdam DB, Donnelly DR. Endophenotypes: a conceptual link between anorexia nervosa and autism spectrum disorder. Res Dev Disabil. 2018;82:153-65.

4 Gillberg C. Are autism and anorexia nervosa related? Br J Psychiatry. 1983;142:428.

5 Baron-Cohen S, Jaffa T, Davies S, Auyeung B, Allison C, Wheelwright S. Do girls with anorexia nervosa have elevated autistic traits? Mol Autism. 2013;4:24.

6 Hambrook D, Tchanturia K, Schmidt U, Russell T, Treasure J. Empathy, systemizing, and autistic traits in anorexia nervosa: a pilot study. Br J Clin Psychol. 2008;47:335-9.

7 Bjornsdotter M, Davidovic M, Karjalainen L, Starck G, Olausson H, Wentz E. Grey matter correlates of autistic traits in women with anorexia nervosa. J Psychiatry Neurosci. 2018;43:79-86.

8 Zucker NL, Losh M, Bulik CM, LaBar KS, Piven J, Pelphrey KA. Anorexia nervosa and autism spectrum disorders: guided investigation of social cognitive endophenotypes. Psychol Bull. 2007;133: 976-1006.

9 Huke V, Turk J, Saeidi S, Kent A, Morgan JF. Autism spectrum disorders in eating disorder populations: a systematic review. Eur Eat Disord Rev. 2013;21:345-51.

10 Westwood H, Eisler I, Mandy W, Leppanen J, Treasure J, Tchanturia $\mathrm{K}$. Using the autism-spectrum quotient to measure autistic traits in anorexia nervosa: a systematic review and meta-analysis. J Autism Dev Disord. 2016;46:964-77.

11 Westwood H, Stahl D, Mandy W, Tchanturia K. The set-shifting profiles of anorexia nervosa and autism spectrum disorder using the Wisconsin Card Sorting Test: a systematic review and meta-analysis. Psychol Med. 2016;46:1809-27.

12 Courty A, Maria AS, Lalanne C, Ringuenet D, Vindreau C, Chevallier C, et al. Levels of autistic traits in anorexia nervosa: a comparative psychometric study. BMC Psychiatry. 2013;13:222.

13 Tchanturia K, Smith E, Weineck F, Fidanboylu E, Kern N, Treasure J, et al. Exploring autistic traits in anorexia: a clinical study. Mol Autism. 2013;4:44.

14 Tchanturia K, Adamson J, Leppanen J, Westwood H. Characteristics of autism spectrum disorder in anorexia nervosa: a naturalistic study in an inpatient treatment programme. Autism. 2019;23:123-30.

15 Nielsen S, Anckarsäter H, Gillberg C, Gillberg C, Råstam M, Wentz E. Effects of autism spectrum disorders on outcome in teenage-onset anorexia nervosa evaluated by the Morgan-Russell outcome assessment schedule: a controlled community-based study. Mol Autism. 2015;6:14.

16 Stewart CS, McEwen FS, Konstantellou A, Eisler I, Simic M. Impact of ASD traits on treatment outcomes of eating disorders in girls. Eur Eat Disord Rev. 2017;25:123-8.

17 Hudson CC, Hall L, Harkness KL. Prevalence of depressive disorders in individuals with autism spectrum disorder: a meta-analysis J Abnorm Child Psychol. 2019;47:165-75.

18 Takara K, Kondo T. Comorbid atypical autistic traits as a potential risk factor for suicide attempts among adult depressed patients: a casecontrol study. Ann Gen Psychiatry. 2014;13:33.

19 American Psychiatric Association. Diagnostic and Statistical Manual of Mental Disorders, Fifth Edition (DSM-5). Arlington: American Psychiatric Publishing; 2013.

20 Vandenbroucke JP, Von Elm E, Altman DG, Gøtzsche PC, Mulrow $\mathrm{CD}$, Pocock SJ, et al. Strengthening the Reporting of Observational Studies in Epidemiology (STROBE): explanation and elaboration. PLoS Med. 2007;4:e297.

21 Ruta L, Mazzone D, Mazzone L, Wheelwright S, Baron-Cohen S. The autism-spectrum quotient--Italian version: a cross-cultural confirmation of the broader autism phenotype. J Autism Dev Disord. 2012; 42:625-33.

22 Hamilton M. A rating scale for depression. J Neurol Neurosurg Psychiatry. 1960;23:56-62.

23 Hamilton $\mathrm{M}$. The assessment of anxiety states by rating. $\mathrm{Br} \mathrm{J}$ Med Psychol. 1959;32:50-5. 
24 Bellace DL, Tesser R, Berthod S, Wisotzke K, Crosby RD, Crow SJ, et al. The Yale-Brown-Cornell eating disorders scale self-report questionnaire: a new, efficient tool for clinicians and researchers. Int $\mathrm{J}$ Eat Disord. 2012;45:856-60.

25 Calugi S, Ricca V, Castellini G, Lo Sauro C, Ruocco A, Chignola E, et al. The eating disorder examination: reliability and validity of the Italian version. 2015;20:505-11.

26 Eisen JL, Phillips KA, Baer L, Beer DA, Atala KD, Rasmussen SA. The brown assessment of beliefs scale: reliability and validity. Am J Psychiatry. 1998;155:102-8.

27 Warrier V, Toro R, Chakrabarti B; iPSYCH-Broad autism group, Børglum AD, Grove J; Me Research Team, et al. Genome-wide analyses of self-reported empathy: correlations with autism, schizophrenia, and anorexia nervosa. Transl Psychiatry. 2018;8:35.

28 Hiller R, Pellicano L. Autism and anorexia: a cautionary note. Psychologist. 2013;26:780.

29 Mandy W, Tchanturia K. Do women with eating disorders who have social and flexibility difficulties really have autism? A case series. Mol Autism. 2015;6.

30 Rizk M, Lalanne C, Berthoz S, Kern L; EVHAN Group, Godart N. Problematic exercise in anorexia nervosa: testing potential risk factors against different definitions. PLoS One. 2015;10:e0143352.

31 Claudino AM, Hay P, Lima MS, Bacaltchuk J, Schmidt U, Treasure J. Antidepressants for anorexia nervosa. Cochrane Database Syst Rev. 2006;(1):CD004365.

32 Malhi GS, Byrow Y, Outhred T, Fritz K. Exclusion of overlapping symptoms in DSM-5 mixed features specifier: heuristic diagnostic and treatment implications. CNS Spectr. 2017;22:126-33.

33 Derks EM, Allardyce J, Boks MP, Vermunt JK, Hijman R, Ophoff RA, et al. Kraepelin was right: a latent class analysis of symptom dimensions in patients and controls. Schizophr Bull. 2012;38: 495-505.

34 Bynum B. Discarded diagnoses monomania. Lancet. 2003;362:1425

35 Fornaro M, Perugi G, Gabrielli F, Prestia D, Mattei C, Vinciguerra V, et al. Lifetime co-morbidity with different subtypes of eating disorders in 148 females with bipolar disorders. J Affect Disord. 2010;121:147-51.

36 Reininghaus EZ, Lackner N, Fellendorf FT, Bengesser S, Birner A, Reininghaus $\mathrm{B}$, et al. Weight cycling in bipolar disorder. J Affect Disord. 2015;171:33-8.

37 Fornaro M, Anastasia A, Novello S, Fusco A, Pariano R, De Berardis $D$, et al. The emergence of loss of efficacy during antidepressant drug treatment for major depressive disorder: an integrative review of evidence, mechanisms, and clinical implications. Pharmacol Res. 2019;139:494-502.

38 Fornaro M, Ventriglio A, De Pasquale C, Pistorio ML, De Berardis D, Cattaneo $\mathrm{Cl}$, et al. Sensation seeking in major depressive patients: relationship to sub-threshold bipolarity and cyclothymic temperament. J Affect Disord. 2013;148:375-83.

39 Fornaro $M$, Giosuè $\mathrm{P}$. Current nosology of treatment resistant depression: a controversy resistant to revision. Clin Pract Epidemiol Ment Health. 2010;6:20-4.

40 Hilbert A, Hoek HW, Schmidt R. Evidence-based clinical guidelines for eating disorders: international comparison. Curr Opin Psychiatry. 2017;30:423-37.

41 Senatore I. Studi sull'anoressia. Roma: Alpes Italia; 2015.

42 Abbate-Daga G, Amianto F, Delsedime N, De-Bacco C, Fassino S. Resistance to treatment and change in anorexia nervosa [corrected]: a clinical overview. BMC Psychiatry. 2013;13:294.

43 Alderson P. Absence of evidence is not evidence of absence. BMJ. 2004;328:476-7. 